\title{
RATIONAL USE OF BLOOD TRANSFUSION DURING OPEN REDUCTION AND INTERNAL FIXATION IN PATIENTS WITH ISOLATED CLOSED FEMORAL FRACTURES AT MULAGO HOSPITAL
}

\author{
C.K.Kinyanjui, MBChB (Moi), MMed (Orth) MUK, P.O. Box 19407-00100, Nairobi, Kenya. Email:ckiarie2002ke@ \\ yahoo.com
}

\section{ABSTRACT}

Background: Blood transfusion can be a life saving intervention. However, if blood is given when it is not needed, the patient receives no benefit and is exposed to unnecessary risk. Therefore, transfusion should be prescribed only when the benefits to the patient are likely to outweigh the risks.

Objective: To evaluate the rationale for blood transfusion in patients undergoing open reduction and internal fixation (ORIF) for isolated closed femoral fractures during the study period in Mulago Hospital.

Design: This was a cross-sectional analytical study.

Setting: Orthopaedic theatre, Mulago National Hospital, Uganda.

Methods: Purposive sampling was used.These were patients in Ward 7 of Mulago Hospital. The allowable blood loss was calculated using the haemodilution method. Blood loss was calculated by weighing dry and blood soaked gauze swabs. The amount of intravenous fluids was recorded. The patient was monitored for pulse rate, blood pressure and urine output. Data processing and analysis was done by use of SPSS-10 package.

Results: Only $55 \%$ of patients required blood by WHO criteria intraoperatively. Of those who actually received blood $35.5 \%$ were irrational.

Conclusion: Physiological monitoring is an effective way of determining which patients require blood transfusion as per the WHO criteria during ORIF of isolated closed femoral fractures. Only $55 \%$ of the blood transfusions were rational as per the WHO criteria during the study period.

\section{INTRODUCTION}

The probability of window period infection for HIV is very high in high prevalence populations. Some degree of blood loss can often be safely incurred before blood transfusion becomes necessary, provided that the loss is replaced with intravenous fluids to maintain normovolemia. However, unexpected blood loss can occur during any type of surgery. The patients $\mathrm{Hb}$, although important, should not be the sole deciding factor in starting a transfusion. The decision should be supported by the need to relieve clinical signs and symptoms and prevent significant morbidity and mortality (1). In trauma patients, blood transfusions have been shown to be an independent risk factor for death, perioperative infection, post injury multiple organ failure, admission to ICU and SIRS (2). For patients in shock, those who underwent blood transfusion were three times more likely to die and greater than three times more likely to be admitted to ICU ( $P=\leq 0.001)$ (2). Admission anemia was found to be an independent predictor ( $P=0.008)$ (2).

In a retrospective cohort study of 9598 consecutive hip fracture patients who underwent repair, blood transfusion was associated with 1.35 fold increased risk of bacterial infection and 1.52 fold increased risk for pneumonia ( $\mathrm{P}=\leq 0.005)$ (2). In an evaluation of 765 transfusion episodes in 560 patients, $42.3 \%$ were found to be inapprorpirate (3). Majority were because of over estimation of immediate risk incurred by withholding the transfusion (3).

\section{MATERIALS AND METHODS}

All patients for ORIF of isolated femoral fracture who were 12 years of age and above who consented or whose guardians consented for the study were thoroughly assessed by the Principle Investigator to rule out any cardio respiratory or any other illness. Preoperative investigations such as ECG, liver function tests and renal function tests were done depending on the age and the clinical condition of the patient. Patients were premedicated with diazepam $10 \mathrm{mg}$ the night before the operation and their baseline blood pressure, respiratory rate and pulse rate taken on the morning of the operation. Patients were weighed on the morning of the operation. Allowable blood loss was calculated.

During the operation, gauze swabs were weighed in their dry state while in their sterile packs. Blood soaked swabs were weighed as soon as they were discarded into a plastic paper bag. The suction bottle was weighed before and after use. This is because the calibration on the suction bottle is widely spaced and hence difficult to quantify small differences in blood loss by visual observation. Any gauze swabs not used were subtracted from the initial dry weight. It has been shown that $1 \mathrm{ml}$ of blood weighs approximately 
1g. This was the difference in weight of the used dry and wet swabs. Volume of irrigation fluids was noted and subtracted from the measured blood loss. Blood loss onto the floor was estimated and added to that of the wet swabs and suction bottle to get the total blood loss. Blood on the floor was estimated at the ratio of one litre of blood spreads over an area of 1 square meter (1). Large blood clots were weighed together with the wet gauze swabs. Gauze mops were spread around the surgical area on the surgical drapes to absorb any blood spillage. The total volume of crystalloid solutions such as normal saline given by the anaesthetist were charted.

Quarter hourly measurements of pulse rate, respiratory rate, blood pressure and urine output, were taken and charted. Special remarks concerning the intraoperative period were noted to explain any complications that occurred either anaesthetic or surgical.

Postoperatively blood loss into the drainage bag was charted for the first postoperative day. Pulse rate, respiratory rate, blood pressure, and urine output $(\mathrm{N}=0.5 \mathrm{ml} / \mathrm{kg} / \mathrm{hr}$ ) was also charted 4 hourly. The patient was asked to report immediately if he got chest pain. Patient's blood for transfusion was ready in theatre before the operation started. Postoperative haematocrit was done on day 1 and $\mathrm{Hb}$ on day 3. HCT is a sensitive measure of the haemoglobin after acute blood loss. The $\mathrm{Hb}$ reflects the true haemoglobin level after the compensatory mechanisms to restore blood volume are completed, usually by the third day.

\section{RESULTS}

For those patients who did not require blood as per the WHO criteria there was no significant change in diastolic BP and PR and this relationship was statistically significant $(p$-value $=0.002)$ (Table 1$)$.

For those patients found to require blood intraoperativelyby theWHOcriteria,therewasasignificant change in diastolic BP and PR and this relationship was statistically significant ( $p$-value $=0.000$ ) (Table 2).

For those patients found not to require blood by the WHO criteria, there was no significant change in BP and PR and this relationship was statistically significant ( $p$-value $=0.000$ ) (Table 3 ).

For those patients who were found to require blood by the WHO criteria postoperatively, there was a significant change in BP and PR and this relationship was statistically significant $(p$-value $=0.000$ ) (Table 4).

Table 1

Vital signs for those not requiring blood intraoperatively by WHO criteria

\begin{tabular}{llllll}
\hline Tests & Number & Mean BP & Std deviation & df & Sig. \\
\hline BP diastolic preoperative & 9 & 84.44 & 10.138 & & \\
BP diastolic intraoperative (Average) & 9 & 80.4120 & 7.98696 & & \\
PR preoperative & 9 & 82.33 & 5.099 & 11.3377 & \\
PR intra operative (Average) & 9 & 96.2642 & & 0.002
\end{tabular}

Table 2

Vital signs for those requiring blood intraoperatively by WHO criteria

\begin{tabular}{llllll}
\hline Tests & Number & Mean BP & Std deviation & df & Sig. \\
\hline BP diastolic preoperative & 31 & 84.35 & 10.062 & & \\
BP diastolic intraoperative (Average) & 31 & 77.3758 & 8.09268 & & \\
PR preoperative & 31 & 80.94 & 16.470 & 30 & 0.000 \\
PR intra operative (Average) & 31 & 96.9982 & 11.608 & & \\
\hline
\end{tabular}

Table 3

Vital signs for those not requiring blood postoperatively

\begin{tabular}{|c|c|c|c|c|c|}
\hline Tests & Number & Mean BP & Std deviation & df & Sig. \\
\hline BP diastolic preoperative & 30 & 83.00 & 10.222 & & \\
\hline BP diastolic intraoperative (Average) & 30 & 78.1524 & 8.22159 & & \\
\hline PR preoperative & 30 & 78.63 & 12.808 & 29 & 0.000 \\
\hline PR intra operative (Average) & 30 & 87.8429 & 7.14161 & & \\
\hline
\end{tabular}


Table 4

Vital signs for those requiring blood postoperatively by the WHO criteria

\begin{tabular}{|c|c|c|c|c|c|}
\hline Tests & Number & Mean BP & Std deviation & df & Sig. \\
\hline BP diastolic preoperative & 10 & 88.50 & 8.182 & & \\
\hline BP diastolic intraoperative (Average) & 10 & 75.8571 & 4.68751 & & \\
\hline PR preoperative & 10 & 89.10 & 17.578 & 9 & 0.000 \\
\hline PR intra operative (Average) & 10 & 93.4714 & 8.85306 & & \\
\hline
\end{tabular}

Only 55\% required blood intraoperatively as per the WHO criteria. Of those transfused 11(35.5\%)patients were irrationally transfused by this criteria (Table 5).

Only 30\% required blood postoperatively as per the WHO criteria. Of those transfused half (50\%) were irrationally transfused (Table 6).

Most patients were starved for 12 hours (Figure 1). The numbers inside the graph represent the actual number of patients starved for that period of hours.

Total fluid replacement for the first 24 hours was much less than that expected by WHO criteria and this was statistically significant ( $p$-value $=0.00$ ) (Table 7).

There was decreased urine output in most patients intraoperatively and postoperatively and this was statistically significant ( $p$-value $=0.013$ ) (Table 8).

Table 5

Transfusion intra-operatively by WHO criteria

\begin{tabular}{llll}
\hline Transfusion intra-operatively & \multicolumn{2}{c}{ WHO transfusion recommended intra-operatively } & Total \\
& Yes & No & $31(100 \%)$ \\
\hline Yes & $20(64.5 \%)$ & $11(35.5 \%)$ & $9(100 \%)$ \\
No & $2(22.2 \%)$ & $7(77.8) \%$ & $40(100 \%)$ \\
\hline Total & $22(55 \%)$ & $18(45 \%)$ & $40 \%$ \\
\hline
\end{tabular}

Table 6

Transfusion post-operatively by WHO criteria

\begin{tabular}{llll}
\hline Transfusion post-operatively & \multicolumn{2}{c}{ WHO transfusion recommended post operatively } & Total \\
& Yes & No & $10(100 \%)$ \\
\hline Yes & $5(50 \%)$ & $23(50 \%)$ & $30(100 \%)$ \\
No & $7(23.3 \%)$ & $28(70 \%)$ & $40(100 \%)$ \\
\hline Total & $12(30 \%)$ & & \\
\hline
\end{tabular}

Figure 1

Period of starvation (hrs) preoperatively

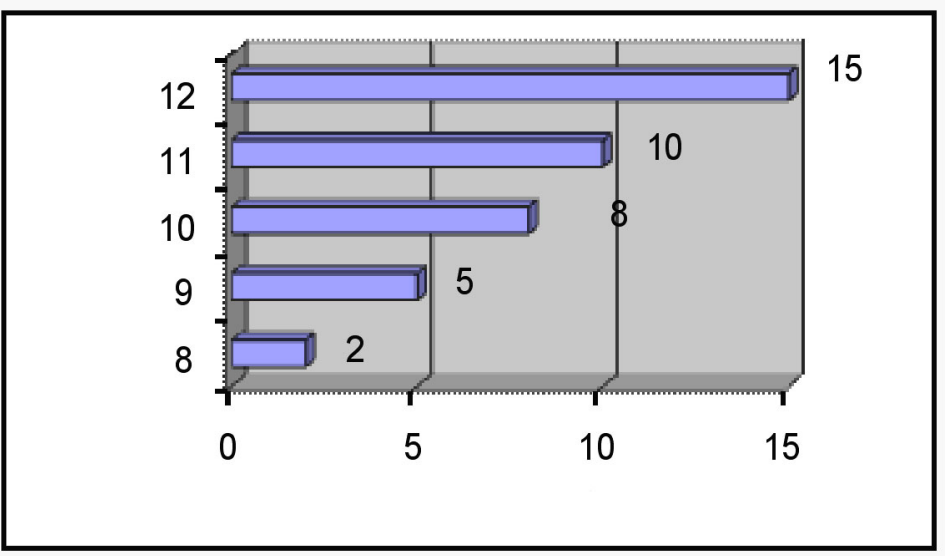


Table 7

Total fluid replacement versus WHO criteria

\begin{tabular}{llllll}
\hline Fluids & Number & Mean fluid & Std deviation & df & Sig. \\
\hline Quantity replaced & 40 & 1712.50 & 587.121 & 39 & 0.00 \\
WHO recommended quantity & 40 & 5520.6563 & 2718.23116 & & \\
\hline
\end{tabular}

Table 8

Urine output versus the expected output intra and post operatively

\begin{tabular}{llllll}
\hline Urine output & Number & Mean urine & Std deviation & df & Sig. \\
\hline Urine output intra-operatively & 40 & 49.38 & 14.021 & & \\
Urine expected intra-operatively & 40 & 56.14 & 21.733 & \multirow{2}{*}{39} & 0.013 \\
Urine output post operatively & 40 & 703.63 & 118.115 & 95.226 & \\
Urine expected post operatively & 40 & 779.40 & & \\
\hline
\end{tabular}

\section{DISCUSSION}

Using the WHO criteria for blood transfusion only 55\% of patients required blood transfusion intra-operatively. Of those transfused, 11 (35.5\%) were irrational by this criteria. This is close to another study done, which revealed that $47 \%$ of transfusions in that particular hospital had no scientific basis (3). They associated those transfusions to the physicians overestimation of the immediate risk of withholding the transfusion(3). In the post operative period $70 \%$ of the patients did not require blood transfusion as per the WHO criteria (Table 6). Among those transfused, half (50\%) were irrational by this criteria.

Among the patients not requiring blood intraoperatively there was no significant change in diastolicbloodpressure ( $p$-value $=0.154$ ). However,there was a significant rise in the pulse rate $(p$-value $=0.002$ ) (Table 1), indicating that diastolic blood pressure is a more reliable indicator of transfusion needs intraoperatively than pulse rate (1). Pulse rate may change depending on the depth of anaesthesia and analgesia (1). For those who were rationally transfused intraoperatively, there was a significant decrease in the diastolic blood pressure ( $p$-value $<0.001$ ), the pulse rate also changed significantly (1) $(p$-value $=0.001)$ (Table 2). Similar findings were observed in those who were transfused rationally in the post operative period (Table 4).

The $\mathrm{Hb}$ on day 3 correlated significantly with preoperative $\mathrm{Hb}$ ( $\mathrm{p}$-value $<0.001)$. It also correlated significantly with intraoperative blood loss (4) ( $p$-value $=0.008$ ). However, its relationship with post-operative blood loss was not significant ( $p$-value $=0.284$ ). The relationship between preoperative $\mathrm{Hb}$ and intraoperative blood loss was not significant $(p$-value $=0.397)$. Thus the preoperative $\mathrm{Hb}$ did not influence the need for transfusion, rather it was the intraoperative blood loss and the drop in diastolic pressure which had a significant relationship with the need for blood transfusion.
Among the nine patients not transfused intraoperatively, two required blood transfusion by WHO criteria (Table 5). There were also seven patients who required blood transfusion postoperatively but who were not transfused (Table 6). These patients later stabilized on intravenous fluids but their $\mathrm{Hb}$ on day 3 was low (mean $8 \mathrm{~g} \mathrm{dl}$ ) This shows lack of a clear criteria in the administration of blood transfusion in patients undergoing ORIF of femur. The rehydration needs per patient also differed significantly with the expected rehydrationas pertheWHOcriteria ( $p$-value $<0.001$ ) (Table 7), this may explain the decreased urine output in most of the patients both intraoperatively and postoperatively (p-value <0.001) (Table 8). Most patients were starved for 12 hours before surgery (37.5\%) (Figure 1).This increased their rehydration needs. It was also noted that most patients routinely received $1500-2000 \mathrm{mls}$ of normal saline despite having different rehydration needs.

\section{CONCLUSION}

Physiological monitoring of patients as per theWHO criteria is an effective way of determining which patients require transfusion during ORIF of femur in Mulago Hospital.

\section{REFERENCES}

1. Jean, C. and Antony, C., (Eds). WHO Blood Transfusion Safety. The clinical use of blood in medicine, obstetrics, paediatrics, surgery and anesthesia, trauma and burns (WHO). 2001; 4:1-426.

2. Debra, L., Malone J., Richard W., et al. Blood transfusion. Independent of shock severity is associated with worse outcome in Trauma. J. Trauma, Injury, infection and Critical Care. 2003; 54: 898-907.

3. Moses, B., Epstein M., Ben-Bassat I., Modan B. and Hackin, H. Evaluation of appropriate use of blood and blood products transfusion using a preset criteria. Transfusion. 1999; 6: 473-476.

4. Lawrence, R., Benjamin, B. and Rappaport, W. Blood loss in patients with isolated femoral fractures.J. Orthopaedic Trauma. 1992; 6: 175-179. 